\title{
Functional Activation Mapping of Scar-Related Ventricular Tachycardia Substrate
}

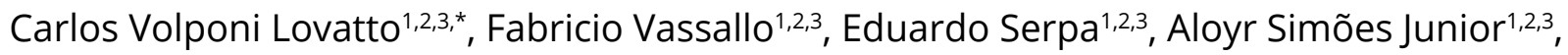
Hermes Carloni i,2,3, Christiano Lemos ${ }^{1,2,3}$, Walter Batista Junior ${ }^{1,2,3}$, Dalton Hespanhol2, Alberto Nogueira², Rafael Zeni ${ }^{4}$, Marcel Burgues 5 , Edevaldo Silva ${ }^{4}$

\section{ORCID IDs}

Lovatto CV (D) https://orcid.org/0000-0002-9225-2875

Lemos C (D) https://orcid.org/0000-0001-5499-4547

vassallo F (D) https://orcid.org/0000-0003-3915-4309

Zeni R (D) https://orcid.org/0000-0002-9602-2559

\begin{abstract}
The optimal method to identify the arrhythmogenic substrate of scar-related ventricular tachycardia (VT) is unknown. Sites of activation slowing during sinus rhythm (SR) often co-localize with the VT circuit. This is a report of two scar related VT substrate mapping using a strategy of voltage-independent approach.
\end{abstract}

KEYWORDS: Ventricular tachycardia; Catheter ablation; Electroanatomical mapping.

\footnotetext{
1. Hospital Unimed, Cardiology - Vitória (ES), Brazil.

2. Hospital Santa Rita, Cardiology - Vitória (ES), Brazil.

3. Hospital Metropolitano, Cardiology - Vitória (ES), Brazil.

4. Abbot Electrophisiology - Vitória (ES), Brazil

5. Boston Scientific Electrophisiology- Vitória (ES), Brazil.

*Correspondonding author: carlos.arritmia@gmail.com

Received: May 22, 2020 | Accepted: Jul 01, 2020
} 


\section{INTRODUCTION}

The primary mechanism of scar-related ventricular tachycardia (VT) is reentry caused by an interplay of the activation and repolarization of the anisotropic properties of the arrhythmogenic substrate ${ }^{1}$.In hemodynamically stable tachycardias, an activation map can identify critical sites of the reentrant circuit that can be targeted with ablation. However, this is rarely accomplished, as the majority of VT are not hemodynamically tolerated ${ }^{1-3}$.

Substrate mapping techniques in sinus rhythm (SR) are an alternative to activation, reducing prolonged periods of mapping during tachycardia ${ }^{1}$. Since the majority of scar-related VT are not hemodynamically tolerated, surrogates in SR for sites critical to reentry are desirable, and late potentials (LP) in SR have become a common ablation target, as they exhibit high sensitivity, albeit with variable specificity ${ }^{2}$.

A commonly used strategy for VT ablation is the tagging and targeting abnormal electrograms (EGM) with local conduction delay on a voltage-based electroanatomical mapping (EAM), and strategies to homogenize scarring by eliminating these abnormal EGM have been shown effective in SR ${ }^{4}$. However, among all identified LP on a substrate, it is not known which zone of late activation most commonly provides the substrate for reentry, suggesting that not all regions of scarring harbor the same potential for arrhythmogenicity ${ }^{3}$.In addition, LP may be unrelated to any VT circuits and may represent unnecessary ablation targets. Therefore, substrate-based ablation of VT has conventionally relied on voltage mapping to delineate low voltage regions, even though it lacks specificity to identify the VT isthmus ${ }^{2-3}$.

Scar dechanneling is a substrate-based strategy performed in sinus or paced rhythm and allows the characterization of scar areas supporting possible VT reentry circuits, named conducting channels (CC), which can be identified by voltage mapping or electrogram analysis and are considered the ablation target. Electrograms with delayed components (E-DC) are tagged and classified as entrance or inner $\mathrm{CC}$ points, depending on delayed-component precocity during $\mathrm{SR}$. The $\mathrm{CC}$ input is defined as the $\mathrm{E}-\mathrm{DC}$ with the shortest delay between the far-field component of healthy muscles (low frequency, usually high voltage) and the local one (delayed, high frequency, usually fractionated and low voltage), corresponding to the local activation of myocardial fibers in the scar. It has been suggested that catheter ablation at the CC entrance can homogenize the scar without extensive ablation ${ }^{5}$.

Long-term follow-up studies have shown smaller recurrence rates with substrate-based approach compared to standard VT ablation ${ }^{6}$. The optimal method to identify the arrhythmogenic substrate of scar-related VT is unknown. Recently, it has been shown that activation sites that slow down during sinus or paced rhythm are often co-localized with the VT circuit $^{1-2}$. Regions of slow conduction isochrones that propagate centripetally into the latest zone of activation may be more specific targets during SR and could be predictive of VT termination sites, providing evidence that deceleration zones (DZ) are highly arrhythmogenic. These data suggest that the latest LP, which represent activation distal to a region of conduction deceleration, may not be essential for reentry maintenance. Rather, deceleration zones exhibiting isochronal crowding may serve as the most specific targets during SR. DZ typically harbors critical isthmus during VT, which may be spatially distinct from the most recent activation region ${ }^{2-3}$.

With the above in mind, we report two cases using a voltage-independent mapping ablation strategy to display targeting regions with isochronal crowding and DZ (functional propagation map), by creating an isochronal late activation mapping (ILAM) .

\section{CASE REPORT}

The first case is that of a 65-year-old male with non-ischemic cardiomyopathy who was admitted to the hospital following more than three episodes of sustained monomorphic VT. He had been treated with an implantable cardioverter defibrillator (ICD) with anti-tachycardia pacing (ATP) and shocks, despite anti-arrhythmic drug therapies (carvedilol and amiodarone). The last therapy converted the first VT into a faster one. Diffuse delayed 
sub-epicardial and mid-wall enhancement in previous cardiac magnetic resonance pointed to a myocarditis etiology. An echocardiogram showed 24\% left ventricle (LV) ejection fraction (EF) and an LV diameter of $69 \mathrm{~mm}$. No obstructions were found on a previous coronary angiography.

The second case is a 63-year-old male diagnosed with heart failure and reduced ejection fraction (LV EF 30\%) secondary to ischemic cardiomyopathy due to multiple previous myocardial infarctions. He was admitted due to palpitations, hypotension, and presyncope followed by ICD shocks, despite anti-arrhythmic drug therapies (metoprolol and amiodarone). Acute myocardial ischemia was ruled out via angiography.

We decided to use VT ablation in both cases, aiming to reduce VT episodes and ICD therapy.

\section{Mapping protocol and ablation}

Depending on the patients' clinical condition, before starting mapping, a VT induction with programmed right ventricle $(\mathrm{RV})$ stimulation was performed and terminated by pacing or cardioversion. The purpose of this step was to determine inducibility and hemodynamic tolerability.

High-density electroanatomic maps were created with multielectrode catheters using EnSite Precision (HD-Grid and 2-2-2 Livewire, Abbott). ILAM was performed during RV pacing rhythm and displayed with eight equally distributed activation isochrones (12.5\% of ventricular activation comprised each isochrone).

An abnormal electrogram was annotated for the offset of local bipolar EGM deflection in real time (nearfield local activation of myocardial fibers in the scar), meaning the completion of local activation to incorporate the latest local activation into the map using ventricular EGM (far-field component of healthy muscle) as a parameter. Isochronal crowding was defined as the local deceleration of propagation in a region with a bunching of isochrones ( $>2$ isochrones within a radius of $1 \mathrm{~cm}$ ). That display allowed us to find and target the first $\mathrm{DZ}$, and not the latest potentials (activation distal to a deceleration conducting region).

In the first patient, the epicardial space through percutaneous subxiphoid access was initially approached with a Tuohy needle and anterior access guided by fluoroscopy (LAO $45^{\circ}$ ) without complications. The mapping and ablation catheter was supported with a non-deflectable sheath (Swartz ${ }^{\mathrm{TM}}$, SL-1 St Jude) and found no resistance in the pericardial space, suggesting an absence of adherences. Using an Advisor ${ }^{\mathrm{TM}} \mathrm{HD}$ Grid SE ${ }^{\mathrm{TM}}$ (Abbott) mapping catheter, areas with abnormal EGM were found only on the epicardial surface, with the $\mathrm{DZ}$ represented by isochronal crowding in a centripetal activation zone (Fig.1).

An epicardial bipolar voltage map revealed a scar area $(<1.5 \mathrm{mV})$ of approximately $44 \%$ of the total area. The endocardial bipolar map showed no abnormal EGM, while the endocardial unipolar map showed low voltage areas (Fig. 2).

These DZ (yellow and orange) were targeted, represented by a centripetal activation, instead of the latest activation zone (purple). Radiofrequency applications were made with an irrigated cool-tip TactiCath SE ${ }^{\mathrm{TM}}$ (Abbott) contact force ablation catheter, aiming an average contact force of $10 \mathrm{~g}$ pointed toward the myocardium with 40 watts and a $30 \mathrm{~mL} / \mathrm{min}$ flow rate. The duration of radiofrequency $(\mathrm{RF})$ application was 14 minutes, and efforts were made to perform epicardial RF ablations with a relatively "dry" pericardial space, to achieve better lesions results. The total procedure time was 3 hours without any complications.

Coronary angiography and phrenic nerve pacing pre-RF applications were done to avoid damage. In the end, we also used an intrapericardial corticosteroid, to avoid pericarditis. Because of the patient's hypotension since the beginning of the procedure, we decided not to try VT induction.

The second case was an LV endocardial-only mapping and ablation. Before mapping, two morphological VT were induced, but they were not hemodynamically tolerated and were interrupted by cardioversion (Fig. 3).

Using a Livewire ${ }^{\mathrm{TM}}$ 2-2-2 Duo-Decapolar (Abbott) mapping catheter, an LV activation map during RV pacing was created and showed a DZ, represented by isochronal crowding in a centripetal activation fashion (Fig. 4). A bipolar voltage map revealed an extensive area of dense scarring, accounting for nearly $45 \%$ of the total area (LV). 


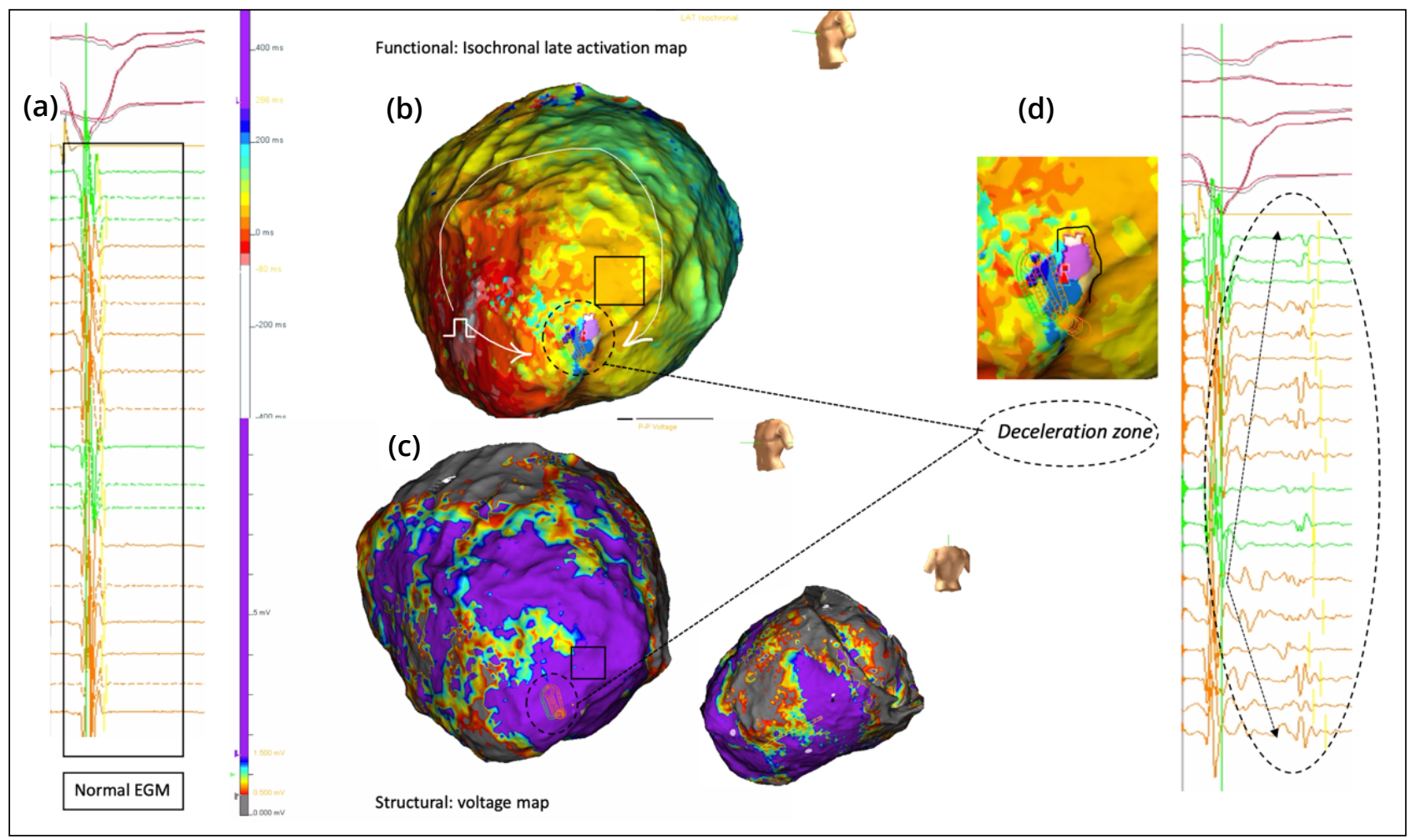

ILAM: isochronal late activation map; RV: right ventricle; DZ: deceleration zone; EGM: electrograms; LV: left ventricular; LAO: left anterior oblique;

Figure 1. High density ILAM with HD grid (Abbott) catheter during RV pacing, demonstrating a DZ due to a small zone of slow conduction. (a) Normal voltage EGM inside the solid square near of the slow conduction area of interest. (b) ILAM of LV epicardium (LAO), demonstrating activation of the LV from anterior (white isochrone earliest) propagating posteriorly across the lateral wall (Ensite Precision, Abbott). The entire window is divided into eight colors of activation (see color bar for sequence), with the latest region of activation represented by the final purple isochrone. Uniform conduction with relatively even isochronal distribution is seen until the interface between the green and light blue isochrones. This discontinuity with isochronal crowding defines a DZ (dashed circle), which is created by a small, localized line of epicardial conduction block (black bold line in the zoomed segment), which forces the activation around the line of the block. (c) Structural voltage map showing an extensive low-voltage area $<1.5 \mathrm{mV}$ (approximately $44 \%$ of total area). DZ localized in a normal voltage area (>1.5mV). (d) Annotation of the latest EGM component (yellow dashed lines) by uniformly selecting the local EGM offset, representing the completion of local activation using ventricular EGM as a reference.

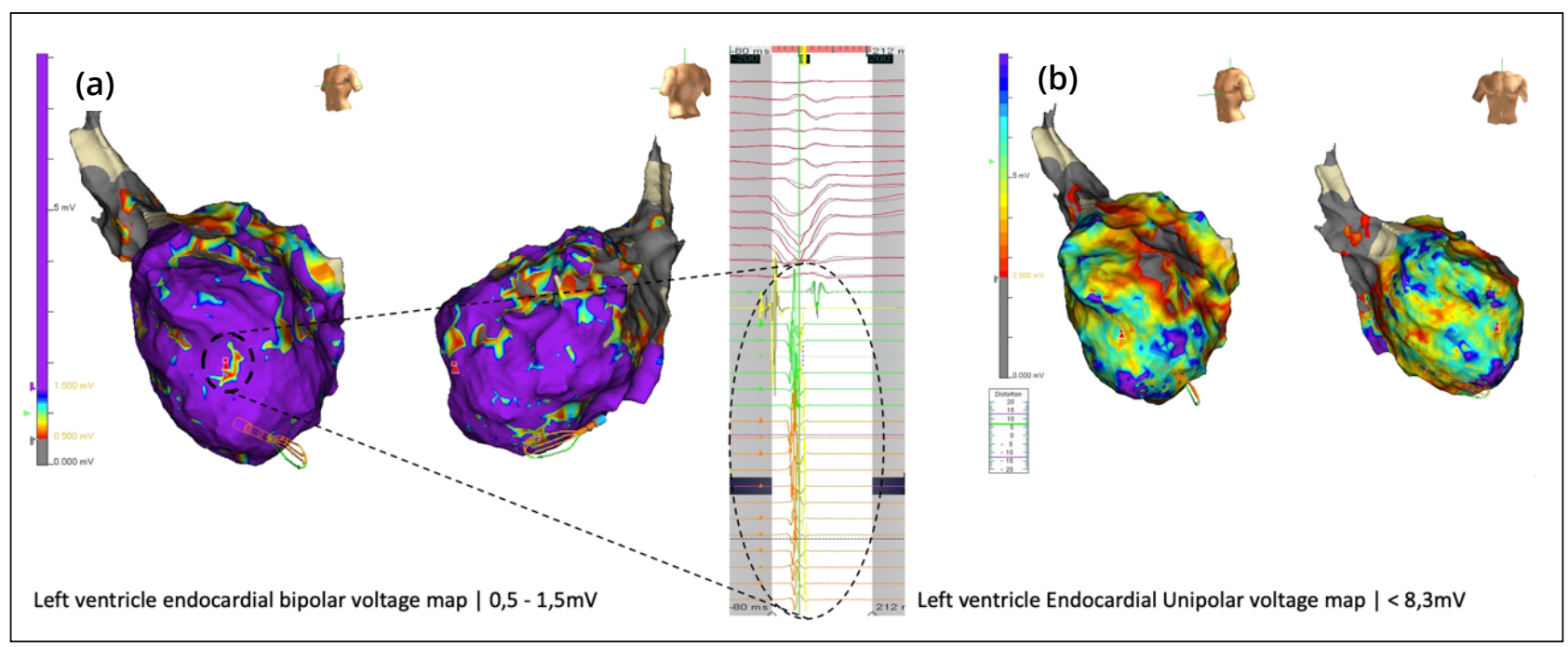

Figure 2. Endocardial voltage map (HD grid). (a) Bipolar voltage map demonstrating normal EGM. Dashed circle shows the corresponding area opposite the epicardial area of interest. (b) Unipolar voltage map demonstrates a low voltage zone, suggesting an epicardial scar. 


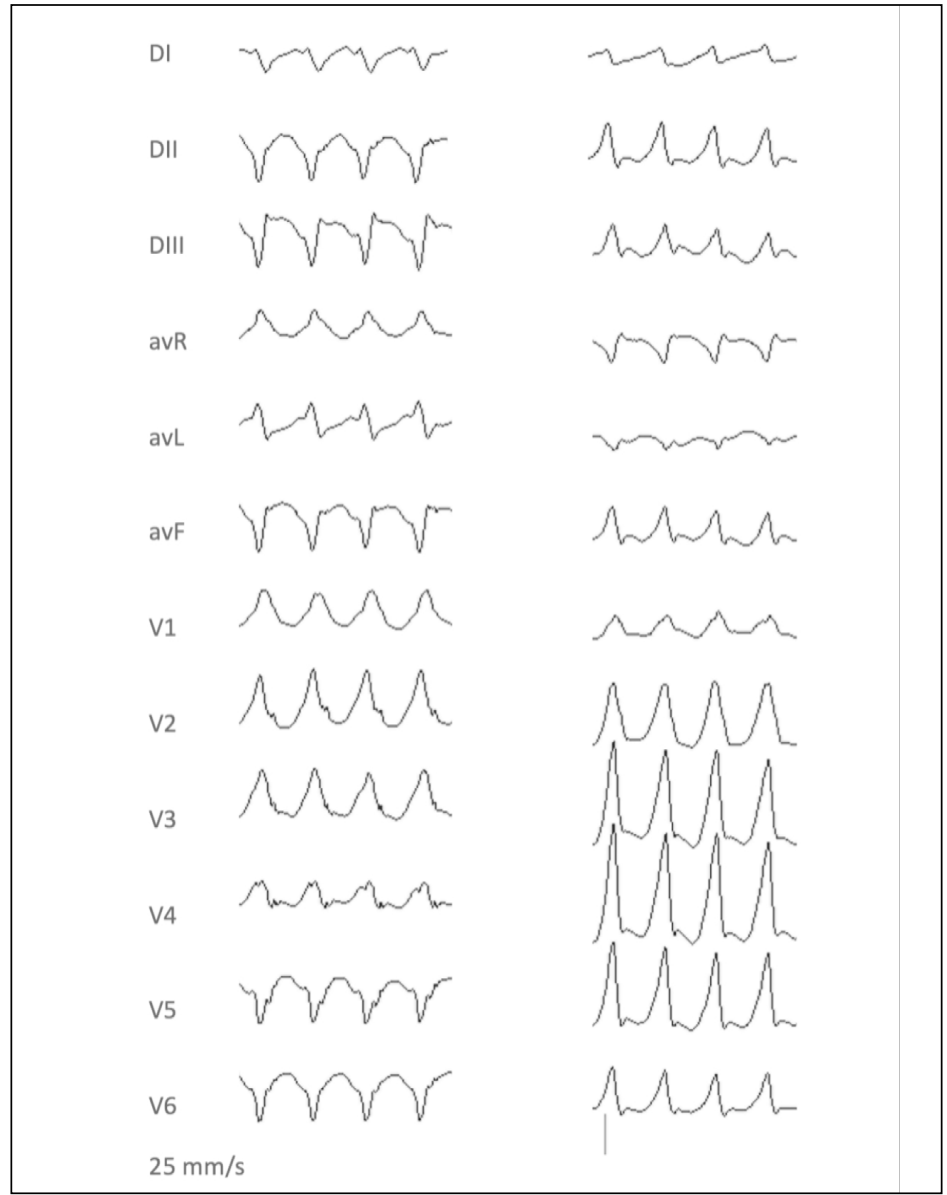

Figure 3. Two morphology ventricular tachycardia induced by ventricular programmed stimulation. Neither were hemodynamically tolerated.

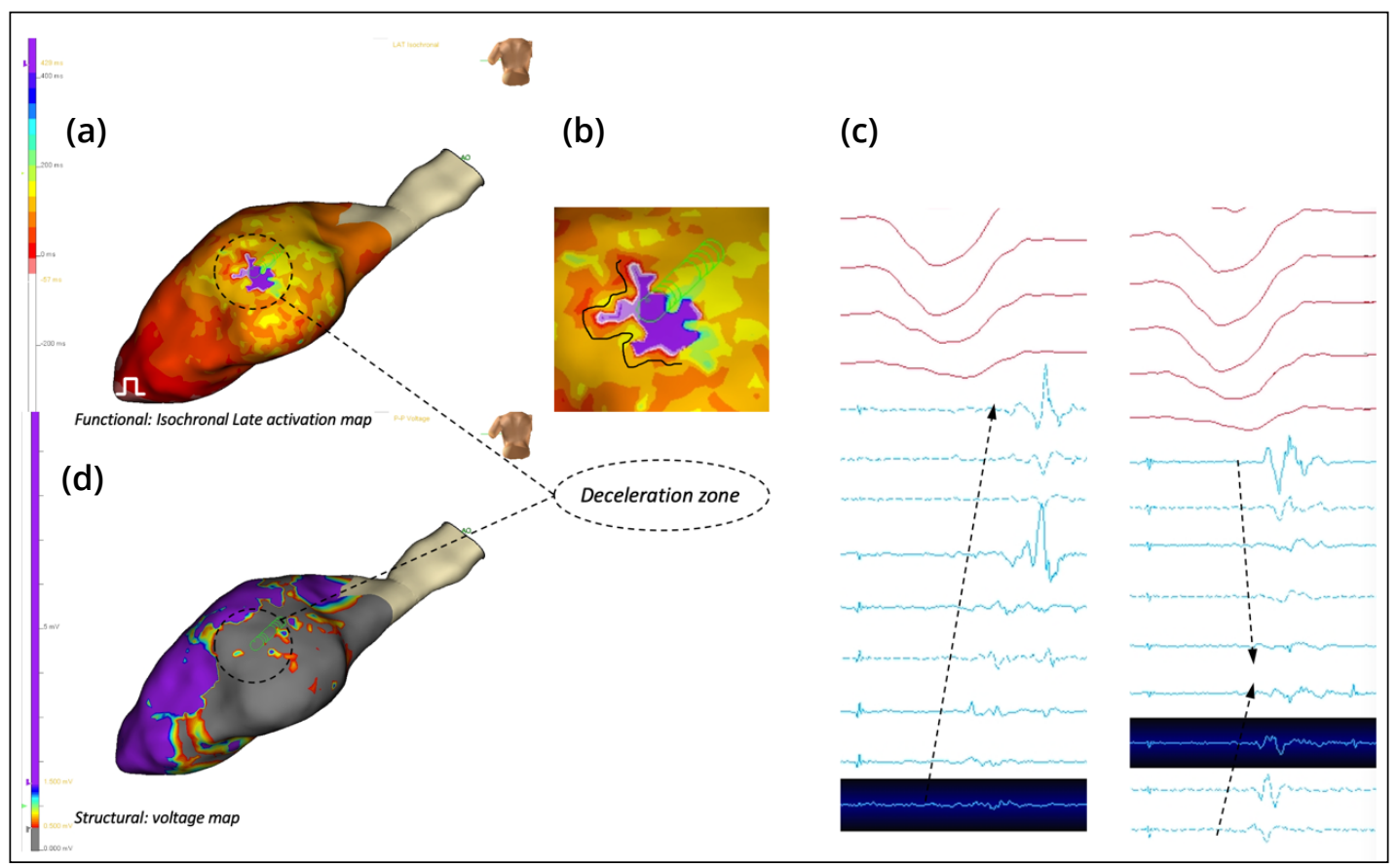

RV: right ventricle; ILAM: isochronal late activation mapping; DZ: deceleration zones.

Figure 4. Left ventricle endocardial map with a Livewire 2-2-2 catheter (Abbott) during RV pacing. (a) ILAM map, demonstrating a DZ and a confluent bunch of colors representing the isochronal crowding (dashed circle). (b) Localized line of conduction block, represented by a black line which forces propagation going around it. (c) Local EGM, suggesting a discontinuous activation, are evidenced by widely split electrograms. (d) Structural voltage map showing an extensive low-voltage area <1.5 mV. DZ localized inside the scar (dashed circle). 
These areas were targeted for RF applications using a FlexAbility ${ }^{\mathrm{TM}}$ (Abbott) irrigated tip ablation catheter with 30 watts and a $30 \mathrm{~mL} / \mathrm{min}$ flow rate. Total $\mathrm{RF}$ time was 25 minutes, and the total procedure time was 3 hours without complications. Programmed ventricular stimulation after ablation did not reinduce any sustained VT.

\section{DISCUSSION}

Scar-related VT ablation is a challenging procedure because of substrate complexity and often because of patients' comorbidities. Due to the frailty of these patients, the potential risk for complications during a procedure and the majority of scar-related VT being hemodynamically untolerated means that surrogates in SR for sites critical to reentry are desirable for a safer and easier ablation.

High-density EAM has become an essential tool for substrate-based ablation of scar-related VT. Structural voltage map displays have served to guide ablation lesion sets targeted with various techniques, such as transecting the scar, encircling the border zone tissue, or homogenizing the scar. Voltage mapping with the goal of identifying critical targets within dense scars or border zones is widely implemented, but has potential pitfalls ${ }^{3}$.Localization of the isthmus by adjusting the voltage limits of bipolar maps demonstrated a specificity of those channels in predicting the location of VT isthmus sites of only $30 \%{ }^{7,8}$. The correlation between voltage amplitude and activation deceleration is complex and, sometimes, low voltage regions exhibit normal activation compared to other areas with normal voltage, which exhibit significant activation deceleration. This shows that the anisotropic properties of the arrhythmogenic substrate are complex and that not all regions of scars harbor the same potential for arrhythmogenicity ${ }^{1}$.

A commonly used strategy for VT ablation is the tagging and targeting of abnormal EGM with local conduction delay on a voltage-based EAM. However, among all identified LP in a given substrate, it is not known which zone of late activation most commonly provides the substrate for reentry and could yield extensive RF applications.

The development of substrate mapping techniques during SR might be an alternative to activation, reducing the need for prolonged mapping periods during tachycardia.

Here, two cases of hemodynamically untolerated sustained VT with large scar areas have been reported. An ablation strategy guided by a voltage-independent functional propagation mapping display to target $\mathrm{DZ}$ were used. Aziz et al. ${ }^{3}$ recently showed a high correlation between $\mathrm{DZ}$ with a centripetal propagation forming an isochronal crowding displayed in an ILAM and critical locations for VT maintenance. We found one area of interest in each case and targeted RF applications obviating the need for extensive ablation. The epicardial map of the first patient demonstrated an area of interest harbored in a normal voltage zone, exhibiting a complex correlation between voltage amplitude and activation deceleration.

Apparently, this strategy helped reducing procedure and RF times, since the scar was very large. Furthermore, mapping during pacing, instead of during VT, rendered a safer procedure.

During a 1-month follow-up after ablation, neither patient had ICD therapies.

One of the limitations of our mapping strategy was mapping during RV pace alone, so we acquired only one wavefront. There is still a debate about this in the literature; for example, while Anter et al. showed that, although the general area of deceleration was independent of the activation wavefront, its precise location and magnitude were influenced by the direction of LV activation. Aziz et al. showed that all DZ were identified and conserved in the same regions in $86 \%$ of cases during multiple activation wavefronts.

\section{CONCLUSION}

A functional propagation voltage-independent mapping strategy during SR may be complementary to voltage-based electroanatomic mapping displays for guiding the functional substrate ablation of scar-related VT. In these patients, the technique helped to reduce the need for extensive RF lesions. 


\section{REFERENCES}

1. Anter E, Neuzil P, Reddy VY, Petru J, Park K, Sroubek J, et al. Ablation of Reentry-Vulnerable-Zones Determined by Left Ventricular Activation from Multiple Directions: A Novel Approach for Ventricular Tachycardia Ablation: A Multicenter Study (PHYSIO-VT). Circ Arrhythm Electrophysiol. 2020;13(6):e008625. https://doi.org/10.1161/CIRCEP.120.008625

2. Irie T, Yu R, Bradfield JS, Vaseghi M, Buch EF, Ajijola O, et al. Relationship Between Sinus Rhythm Late Activation Zones and Critical Sites for Scar-Related Ventricular Tachycardia: systematic analysis of isochronal late activation mapping. Circ Arrhythm Electrophysiol. 2015;8(2):390-9. https://doi.org/10.1161/CIRCEP.114.002637

3. Aziz Z, Shatz D, Raiman M, Upadhyay GA, Beaser AD, Besser SA, et al. Targeted Ablation of Ventricular Tachycardia Guided by Wavefront Discontinuities During Sinus Rhythm: A New Functional Substrate Mapping Strategy. Circulation. 2019;140(17):1383-97. https://doi.org/10.1161/CIRCULATIONAHA.119.042423

4. Di Biase L, Santangeli P, Burkhardt DJ, Bai R, Mohanty P, Carbucicchio C, et al. Endo-Epicardial Homogenization of the Scar Versus Limited Substrate Ablation for the Treatment of Electrical Storms in Patients with Ischemic Cardiomyopathy. J Am Coll Cardiol. 2012;60(2):132-41. https://doi.org/10.1016/j.jacc.2012.03.044

5. Berruezo A, Fernández-Armenta J, Andreu D, Penela D, Herczku C, Evertz R, et al. Scar Dechanneling: New Method for ScarRelated Left Ventricular Tachycardia Substrate Ablation. Circ Arrhythm Electrophysiol. 2015;8(2):326-36. https://doi.org/10.1161/ CIRCEP.114.002386

6. Briceño DF, Romero J, Villablanca PA, Londoño A, Diaz JC, Maraj I, et al. Long-term outcomes of different ablation strategies for ventricular tachycardia in patients with structural heart disease: systematic review and meta-analysis. Europace. 2018;20(1):104-15. https://doi.org/10.1093/europace/eux109

7. Mountantonakis SE, Park RE, Frankel DS, Hutchinson MD, Dixit S, Cooper J, et al. Relationship Between Voltage Map "Channels" and the Location of Critical Isthmus Sites in Patients with Post-Infarction Cardiomyopathy and Ventricular Tachycardia. J Am Coll Cardiol. 2013;61(20):2088-95. https://doi.org/10.1016/j.jacc.2013.02.031

8. Arenal A, del Castillo S, Gonzalez-Torrecilla E, Atienza F, Ortiz M, Jimenez J, Puchol A, et al. Tachycardia-related channel in the scar tissue in patients with sustained monomorphic ventricular tachycardias: influence of the voltage scar definition. Circulation. 2004;110(17):2568-74. https://doi.org/10.1161/01.CIR.0000145544.35565.47 